\title{
Intraoperative Functional Mapping and Monitoring during Glioma Surgery
}

\author{
Taiichi SAITO, ${ }^{1,2}$ Yoshihiro MURAGAKI, ${ }^{1,3}$ Takashi MARUYAMA, ${ }^{1,3}$ \\ Manabu TAMURA, ${ }^{1,3}$ Masayuki NITTA, ${ }^{1,3}$ and Yoshikazu OKADA ${ }^{1}$
}

${ }^{1}$ Department of Neurosurgery and ${ }^{3}$ Faculty of Advanced Techno-Surgery, Institute of Advanced Biomedical Engineering and Science, Tokyo Women's Medical University, Tokyo; ${ }^{2}$ Department of Neurosurgery, Tokyo Rosai Hospital, Tokyo

\begin{abstract}
Glioma surgery represents a significant advance with respect to improving resection rates using new surgical techniques, including intraoperative functional mapping, monitoring, and imaging. Functional mapping under awake craniotomy can be used to detect individual eloquent tissues of speech and/or motor functions in order to prevent unexpected deficits and promote extensive resection. In addition, monitoring the patient's neurological findings during resection is also very useful for maximizing the removal rate and minimizing deficits by alarming that the touched area is close to eloquent regions and fibers. Assessing several types of evoked potentials, including motor evoked potentials (MEPs), sensory evoked potentials (SEPs) and visual evoked potentials (VEPs), is also helpful for performing surgical monitoring in patients under general anesthesia (GA). We herein review the utility of intraoperative mapping and monitoring the assessment of neurological findings, with a particular focus on speech and the motor function, in patients undergoing glioma surgery.
\end{abstract}

Key words: awake craniotomy, functional mapping, cortico-cortical evoked potential, plasticity, motor evoked potentials

\section{Introduction}

In recent years, evidence has accumulated regarding the outcomes of glioma treatment, including improved treatment results with temozolomide (TMZ) and the contribution of molecular markers, such as IDH1 and $1 \mathrm{p} 19 \mathrm{q}$ loss of heterozygosity. ${ }^{1,2)}$ However, subanalyses of recent randomized controlled trials of the effectiveness of chemotherapy in treating World Health Organization (WHO) grade 2 and 3 gliomas have demonstrated that the resection rate is more significantly correlated with the survival time. ${ }^{3,4)}$ In 2001, Lacroix et al. reported that removing 98\% or more of the brain magnetic resonance imaging (MRI) gadolinium-enhanced portion of the tumor was necessary to achieve a significant increase in patient survival among 416 patients with glioblastoma. ${ }^{5)} \mathrm{A}$ decade later, Sanai et al. expanded on this conclusion and demonstrated the clinical value of the extent of resection (EOR) of total and subtotal procedures on a percentile-by-percentile basis. Their analysis

Received January 13, 2014; Accepted August 11, 2014 of 500 consecutive newly diagnosed patients with glioblastoma demonstrated that an EOR of $78 \%$ is the minimum procedure associated with a survival benefit and that this value increases in association with more complete surgical resection (95\%, $98 \%$ or more, and $100 \%$ respectively). ${ }^{6}$ Regarding low-grade gliomas, Smith et al. demonstrated that among 216 patients, those treated with an EOR of at least $90 \%$ had 5- and 8-year overall survival (OS) rates of $97 \%$ and $91 \%$, respectively, whereas those who received an EOR of less than $90 \%$ had 5 - and 8-year OS rates of $76 \%$ and $60 \%$, respectively. ${ }^{7)}$ Recently, we also investigated whether the EOR was associated with progression-free survival (PFS) and OS in 153 patients with low-grade glioma and demonstrated that both the PFS and OS were significantly longer in the patients with an EOR of $90 \%$ or more. ${ }^{8)}$ These results validate the use of a therapeutic strategy aiming for an EOR of $90 \%$ or more in patients with low-grade gliomas.

On the other hand, aggressive resection has the potential to increase the incidence of neurological complications, especially those associated with 
regions within or near eloquent language and motor areas. In order to preserve the neurological function in patients undergoing glioma surgery, various intraoperative neurological monitoring have been developed, including motor evoked potentials (MEPs) ${ }^{9-11)}$ to detect deterioration of the motor function and sensory evoked potentials (SEPs) ${ }^{12,13)}$ to identify the Rolandic cortex. With respect to preserving the language function, awake craniotomy (AC) is a reliable method for ensuring neural integrity during the excision of lesions located within or near eloquent language areas. ${ }^{14)}$ It is therefore possible to electrically stimulate the cortex and subcortical structure in order to locate functional areas with the patient awake. Furthermore, continuous clinical neurological testing can theoretically be used to detect early deficits in the motor, sensory, or language domains during tumor removal. Intraoperative functional mapping and monitoring thus play important roles in glioma surgery in terms of maximizing the EOR while minimizing the risk of neurological morbidity.

\section{Awake Craniotomy}

Functional mapping under AC offers the potential to accurately localize eloquent brain areas. This procedure allows the surgeon to clearly define language, positive motor, and negative motor areas ${ }^{15}$ ) as well as the position of white matter fibers connected with the speech and motor functions, which helps to prevent unexpected neurological complications. A recent meta-analysis including 90 reports of 8,091 adult patients who underwent resection of supratentorial infiltrative glioma with or without intraoperative stimulation mapping (ISM) demonstrated late severe neurological deficits in $3.4 \%$ of the patients who underwent resection with ISM and $8.2 \%$ of the patients who underwent resection without ISM. ${ }^{16)}$ The percentage of radiologically confirmed gross total resection was $75 \%$ [95\% confidence interval (CI), 66\% to $82 \%]$ in the patients treated with ISM and $58 \%$ (95\% CI, $48 \%$ to $69 \%$ ) in those treated without ISM. Most recently, Brown et al. reviewed eight studies comparing the outcomes of craniotomy for tumor resection under general anesthesia (GA) and AC in 951 patients (411 treated with AC and 540 treated with GA). ${ }^{17)}$ The authors' interpretation of the literature suggests that the mean EOR under awake conditions $(41 \%, \mathrm{n}=321)$ is similar to that of GA $(44 \%, n=444)$, while the incidence of postoperative deficits is lower under awake conditions $(7 \%, \mathrm{n}=411)$ versus GA $(23 \%, \mathrm{n}=$ 520). Given the effectiveness of AC for resecting eloquent tumors, these data suggest an expanded role for AC in brain tumor surgery, regardless of the tumor location, indicating that ISM should be universally implemented as standard of care for glioma surgery. AC is recommended according to guidelines for the management of low-grade glioma in Europe (Class III level). ${ }^{3)}$ In Japan, "The Guidelines for Awake Craniotomy" were formulated by the Japan Awake Surgery Conference, and AC has since become the basic procedure for performing resection of tumors within or near eloquent areas. ${ }^{18}$

\section{Language mapping}

The purpose of language mapping is to identify language areas and prevent permanent postoperative language dysfunction by preserving these areas. An efficient method is required for intraoperative language mapping to reduce the time necessary to intraoperatively localize eloquent cortical areas. In order to identify language-related areas preoperatively and shorten the time needed to detect language areas intraoperatively, we actively apply the results of functional MRI using a picturesentence matching task (with active, passive, and scrambled sentences). ${ }^{19)}$ Our intraoperative mapping procedure follows the dedicated guidelines of the Japan Awake Surgery Conference. ${ }^{18)}$ For intraoperative brain mapping, electrical stimulation of the cortex is applied with a repetitive square wave biphasic current of alternating polarity (pulse width: 0.5 msec, frequency: $50 \mathrm{~Hz}$, duration: 1-2 seconds) using a bipolar electrode probe. The continuous digital electrocorticogram (ECoG) activity is monitored after discharge, and to detect seizures. The stimulus intensity is then increased steadily from $2 \mathrm{~mA}$ in a bipolar fashion using stepwise increments of $1 \mathrm{~mA}$ until the effect is obtained or abnormalities are noted on ECoG. The maximum stimulus intensity is $8 \mathrm{~mA}$, which corresponds to $16 \mathrm{~mA}$ if a monophasic pulse is used, in concert with the experience and recommendations of others. ${ }^{18,20-23)}$ The dedicated intraoperative examination monitor for awake craniotomy (IEMAS) is used to demonstrate the language assessment tasks of the patient. ${ }^{24,25)}$ This device allows the real-time visualization, integration, and recording of a wide spectrum of data, including a view of the patient's face during the response, the type of test provided, the position of the cortical stimulator in the surgical field, the anatomical data obtained from the real-time updated neuronavigation system, ${ }^{26)}$ the bispectral index (BIS) monitor, and so forth (Fig. 1).

With the patient counting (from 1 to 30 ), we first check for sites of speech arrest and delay. Stimulation is then performed in a systematic manner every 


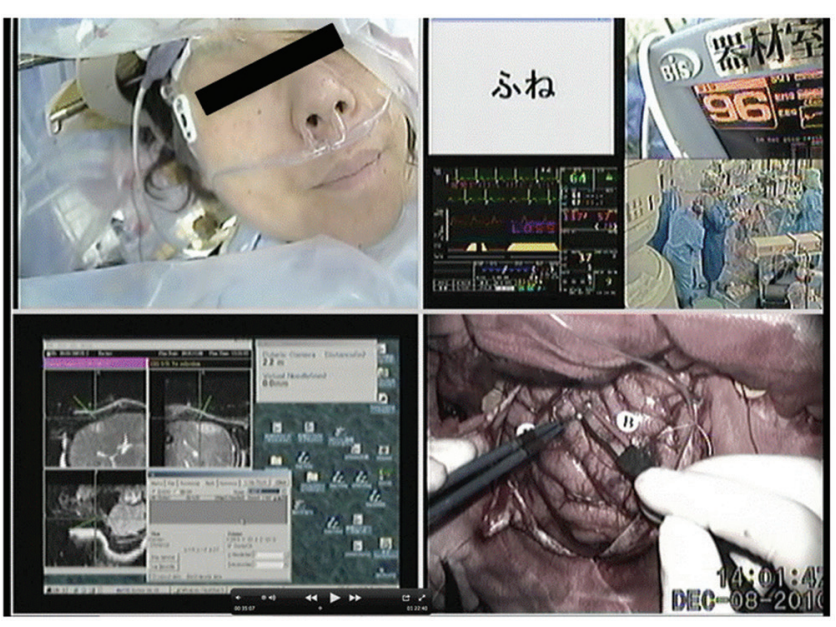

Fig. 1 The screen of the dedicated intraoperative examination monitor for awake craniotomy (IEMAS). Upper left display: The patient's face. Lower left display: The anatomical data obtained from the real-time updated neuronavigation system, which can be used to localize the exact position of the stimulator. Upper right display: Four different sets of data for the test object, bispectral index monitor, heart beat monitor, and general view of the operating theater. Lower right display: The view of the surgical field through the operative microscope.

8-10 $\mathrm{mm}$ along the cortical surface. We subsequently reconfirm the locations of language-related sites using tasks of picture naming, pronouncing written familiar Japanese words, and generating action verbs. During the language mapping procedure, it is important to distinguish the language area from positive (defined as involuntary contractions of the tongue) and negative (defined as impairment of rapid alternating movements of the tongue; arrest of synergic movement) motor areas. After identifying the language areas, we remove the tumor preserving these cortical regions, with subcortical stimulation via the resection cavity directed at clarifying language pathways. The devices used for subcortical stimulation and the parameters of stimulation, including intensity, are similar to those used for cortical mapping. When subcortical stimulation interrupts or disturbs the patient's ability to name a pictured object, the removal procedure is stopped, as we previously confirmed that the responding sites are located very close to the eloquent subcortical fibers (5 mm or less). ${ }^{27)}$ More recently, Trinh et al. ${ }^{28)}$ reported that intraoperative language dysfunction acquired during subcortical dissection is an independent predictor of postoperative deficits in both the immediate postoperative period $(\mathrm{P}<0.001)$ and at the 3 -month follow-up ( $\mathrm{P}$ $<0.001$ ), as observed in 214 patients undergoing AC. Under awake conditions, the patient's ability to speak freely is constantly monitored during the entire procedure with a continuous conversation with a member of the treatment team specialized in assessing the language function who provides specific tasks to evaluate recall, counting, fluency, and comprehension. ${ }^{29)}$

Sanai et al. examined 250 patients with gliomas in order to study the language function after brain-tumor resection using language mapping. ${ }^{23)}$ Cumulatively, 3,281 cortical sites were stimulated in all patients. A total of 145 of the 250 patients $(58.0 \%)$ had at least one site with intraoperative stimulation-induced speech arrest, 82 patients had anomia, and 23 patients had alexia. The authors proposed that the lesion can be removed if stimulation with an intensity of $6 \mathrm{~mA}$ (using a bipolar electrode, $60 \mathrm{~Hz}$, square wave) elicit no speech arrest or delay, which is called "negative mapping" strategy. Surprisingly, they reported that only $1.6 \%$ of surviving patients had a persistent language deficit. In addition, using this strategy, the researchers demonstrated that delineating true functional and nonfunctional areas using intraoperative mapping (in presumed eloquent areas) in high-risk patients to maximize tumor resection dramatically improved the long-term survival of 281 patients with supratentorial lowgrade gliomas. However, it should be kept in mind that this strategy was employed in an experienced facility. Therefore, the use of a "positive mapping" strategy, meaning that the tumor is removed after identifying the language areas, is recommended in less experienced facilities.

\section{Language monitoring}

We consider continuous observation of the patient's intraoperative condition under AC itself to be the most reliable and direct method for monitoring the neurological function. This technique can be used to detect early deficits in the motor, sensory, language, or even higher-order brain functions (e.g., left-right disorientation, acalculia). In addition, continuous direct observation under awake conditions may help the clinician to recognize unexpected neurological complications (e.g., disturbed consciousness due to hemorrhage outside the operative field) in rare cases, thus leading to further imaging studies (e.g., intraoperative MRI or computed tomography), which is useful from the viewpoint of risk management. Importantly, the direct clinical assessments with AC do not show false-negative results, which prevent suboptimal resection in patients undergoing glioma surgery. Consequently, the parallel use of AC and intraoperative neurophysiological monitoring result in more accurate evaluations of the motor and language functions. 


\section{MEPs during AC}

The need for AC to treat gliomas located within or adjacent to the motor eloquent structure is controversial due to differences in the accuracy of MEP monitoring and the goal with respect to the EOR. We actively introduced the use of AC for gliomas located in these areas and have resected such lesions by combining MEP monitoring elicited by direct cortical and transcranial stimulation. ${ }^{30)} \mathrm{We}$ apply MEP monitoring with AC to all patients with glioma located in these areas who have indication for AC recommended by "The Guidelines for Awake Craniotomy" in Japan (e.g., patients aged from 15 years to 65 years). ${ }^{18)}$ On the contrary, we apply MEP monitoring under GA to the patients who do not have indication for AC. The advantages of AC for tumors located in the primary motor cortex or related subcortical fibers is that the surgeon is able to observe the correlations between the results of MEP monitoring, intraoperative voluntary movements, and the involuntary movements elicited by electrical stimulation. Prior studies have reported discrepancies between the results of MEP monitoring and the postoperative or intraoperative neurological status (false-negative and false-positive monitoring). ${ }^{10,11,31 \text { ) }}$ Under awake conditions, even if intraoperative MEP is decreased or absent due to various causes, it is possible to confirm voluntary movements and avoid under-resection. Furthermore, combining MEP monitoring with direct subcortical stimulation and the observation of voluntary movements helps to conduct more accurate evaluations of the intraoperative motor function.

\section{Cortico-Cortical Evoked Potentials (CCEPs)}

Despite the proven effectiveness and widespread acceptance of direct intraoperative brain mapping using electrical stimulation to evaluate the intraoperative language function, the procedure has some limitations. In particular, intraoperative assessments of the verbal response are generally subjective and may be significantly influenced by the patient's level of consciousness and cooperativeness, as well as the parameters of cortical stimulation. In addition, despite the high level of reliability of intraoperative cortical mapping for determining the anatomical localization of language-related areas, the functional interconnections between these regions generally remain obscure. Therefore, the development of further novel methods to objectively assess the language function during $\mathrm{AC}$ is warranted. The technique of cortico-cortical evoked potential (CCEP) monitoring is based on the electrical stimulation of one cortical area while recording the average response from another, which permits the assessment of functional interconnections. ${ }^{32-37)}$

Recently, we and Yamao et al. evaluated recordings of intraoperative CCEP as an adjunctive method for assessing the speech function during resection of intraparenchymal brain neoplasms. ${ }^{38,39)}$ In our report, intraoperative monitoring with CCEP was applied in 13 patients (mean age: $34 \pm 14$ years) during the removal of neoplasms in the dominant cerebral hemisphere located within or around language-related structures. ${ }^{38)}$ For this purpose, strip electrodes were positioned above the frontal language area (FLA) and temporal language area (TLA), which were identified using direct cortical stimulation and/ or preliminary mapping with implanted chronic subdural grid electrodes (Fig. 2). No intraoperative CCEP responses were obtained in one case due to technical problems. In the remaining patients, such responses were identified from the FLA during stimulation of the TLA (seven cases) and from the TLA during stimulation of the FLA (five cases), with a mean peak latency of $83 \pm 15 \mathrm{~ms}$. The CCEP responses well correlated with the postoperative language function, and the time to recovery of the speech function was significantly associated with

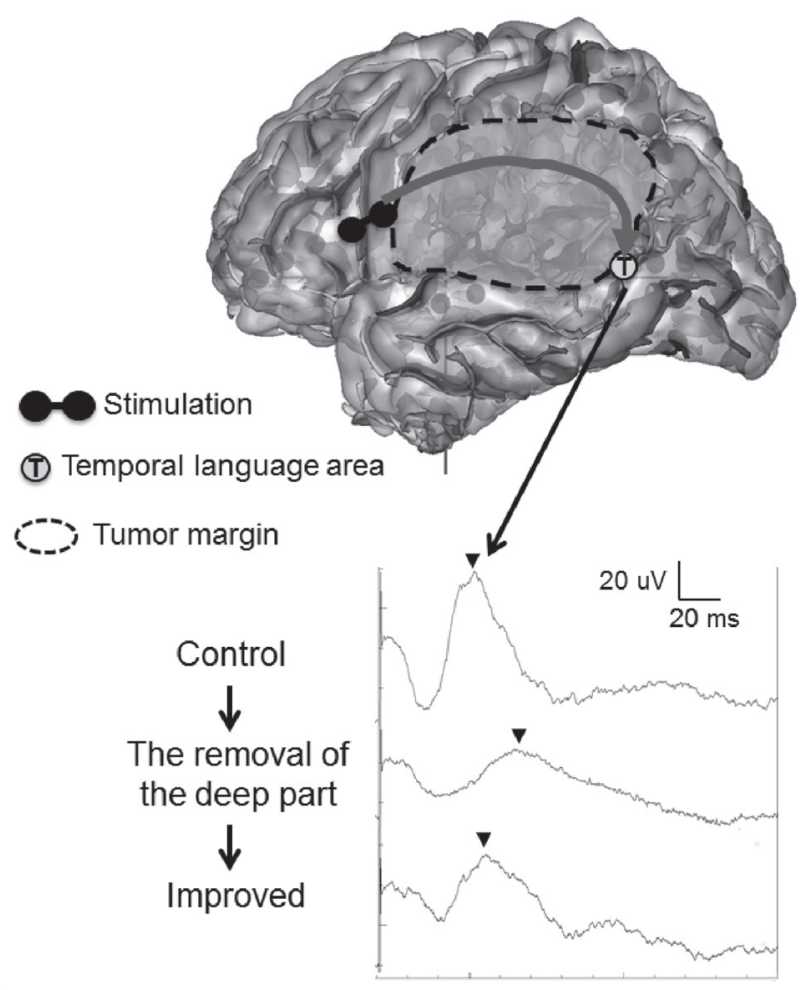

Fig. 2 Intraoperative CCEP monitoring. Changes in CCEP were recognized following stimulation of the frontal language area and recordings of the temporal language area. CCEP: cortico-cortical evoked potential. 
the type of intraoperative change on CCEP, specifically unchanged, decreased, or disappeared during resection at $1.8 \pm 1.0$ months, $5.5 \pm 1.0$ months, and $11.0 \pm 3.6$ months on average, respectively ( $\mathrm{P}$ $<0.01$ ). Remarkably, CCEP recordings are task-free and do not require the cooperation of the patient, ${ }^{37}$ which opens the possibility for monitoring the speech function during neurosurgical procedures performed under GA.

\section{Intraoperative Neurophysiological Monitoring under GA (Particularly MEPs)}

The intraoperative monitoring neurological findings obtained during glioma resection under GA are also useful for maximizing the removal rate and minimizing neurological deficits by alarming that the touched area is close to eloquent regions and fibers. Currently, several types of evoked potentials, including MEPs, SEPs, and visual evoked potentials (VEPs), can be assessed during surgical monitoring under GA. In this article, we primarily review the role of intraoperative MEP monitoring for glioma surgery close to motor areas and/or the pyramidal tract.

Direct electrical cortical and subcortical stimulation is the gold standard for localizing and monitoring the motor function, and this readily available intraoperative technique helps to preserve the eloquent structures of the primary motor cortex and pyramidal tract. ${ }^{40-43)}$ Various stimulus parameters of MEP monitoring for glioma surgery have been reported from different facilities, as shown in Table 1 (e.g., the intensity and threshold of deterioration compared to control values). After placing the strip electrode, the median nerve is first stimulated and the central sulcus is identified based on SEP phase reversal. ${ }^{13)}$ Continuous MEP monitoring using direct cortical stimulation (DCS) with a strip electrode enables a real-time evaluation of the functional integrity of the pyramidal tract. ${ }^{44,45)}$ Meanwhile, intermittent subcortical mapping of the pyramidal tract with a monopolar or bipolar probe can be used to localize pyramidal tracts in white matter. ${ }^{20,46)}$ The predictive value of signal alterations (amplitude and threshold) of MEP monitoring for motor deficits has been demonstrated in several studies. ${ }^{9-11,42,47)}$

\section{MEP monitoring with direct cortical stimulation under GA}

Continuous MEP monitoring of the contralateral upper and lower limb muscle activity using DCS with a strip electrode is widely employed and has been shown to improve the safety of motor eloquent tumor resection. ${ }^{10,42,48-52)}$ In 1993, Taniguchi et al. first reported that intraoperative anodal monopolar cortical stimulation causes compound muscle action potentials (CMAPs) in the upper limbs in patients undergoing human glioma surgery. ${ }^{50)}$ Subsequently, the authors demonstrated that the intraoperative CMAP changes correlate with the postoperative motor function in subjects undergoing brain tumor surgery. ${ }^{53)}$ However, the impact of CMAP monitoring during glioma surgery had not yet been established. The main reason for criticism was that there were no data regarding the effects of monitoring procedures on the rate of resection of gliomas. Because the resection rate correlates with the survival time, as described above, it is of paramount importance to determine the availability of this procedure. On the other hand, Kombos et al. demonstrated that the resection rate and functional outcomes of tumor resection were not negatively influenced by intraoperative CMAP monitoring in 40 patients with glioma. ${ }^{49)}$ Recently, Krieg et al. examined 115 cases of supratentorial glioma located in or close to eloquent motor areas using monitoring of MEP elicited by DCS and found MEP monitoring to be successful in 112 cases (97.4\%). This high success rate confirms that the method is very applicable. The authors focused on cases of false-negative results on intraoperative MEP monitoring. In $65.2 \%$ of the cases, the MEP were stable throughout surgery, although $8.9 \%$ of the patients developed new temporary motor deficits and $4.5 \%$ (five) of the patients presented with permanent deteriorations in the motor function representing false-negative monitoring findings. However, these cases were the result of secondary hemorrhage, ischemia, or resection of supplementary motor areas. The authors therefore concluded that continuous MEP monitoring provides reliable information for the motor system, with no false-negative MEP results. Most recently, Gempt et al. aimed to assess the incidence of resectionrelated ischemia in cases of newly diagnosed or recurrent supratentorial gliomas and determine the sensitivity of intraoperative neuromonitoring of MEPs for detecting such ischemic events and their influence on the neurological motor function in 70 patients with tumors in eloquent motor areas. Nine $(69 \%)$ of 13 patients with a permanent loss of MEP amplitude exhibited postoperative ischemic lesions. The authors warned that,rather than cortical or subcortical structural damage to eloquent brain tissue alone, the development of peri- and/or postoperative ischemic lesions plays a crucial role in the pathogenesis of surgery-related motor deficits. Based on the findings of several clinical series and recently published data, warning criteria have become more refined based on the addition of a decrement 


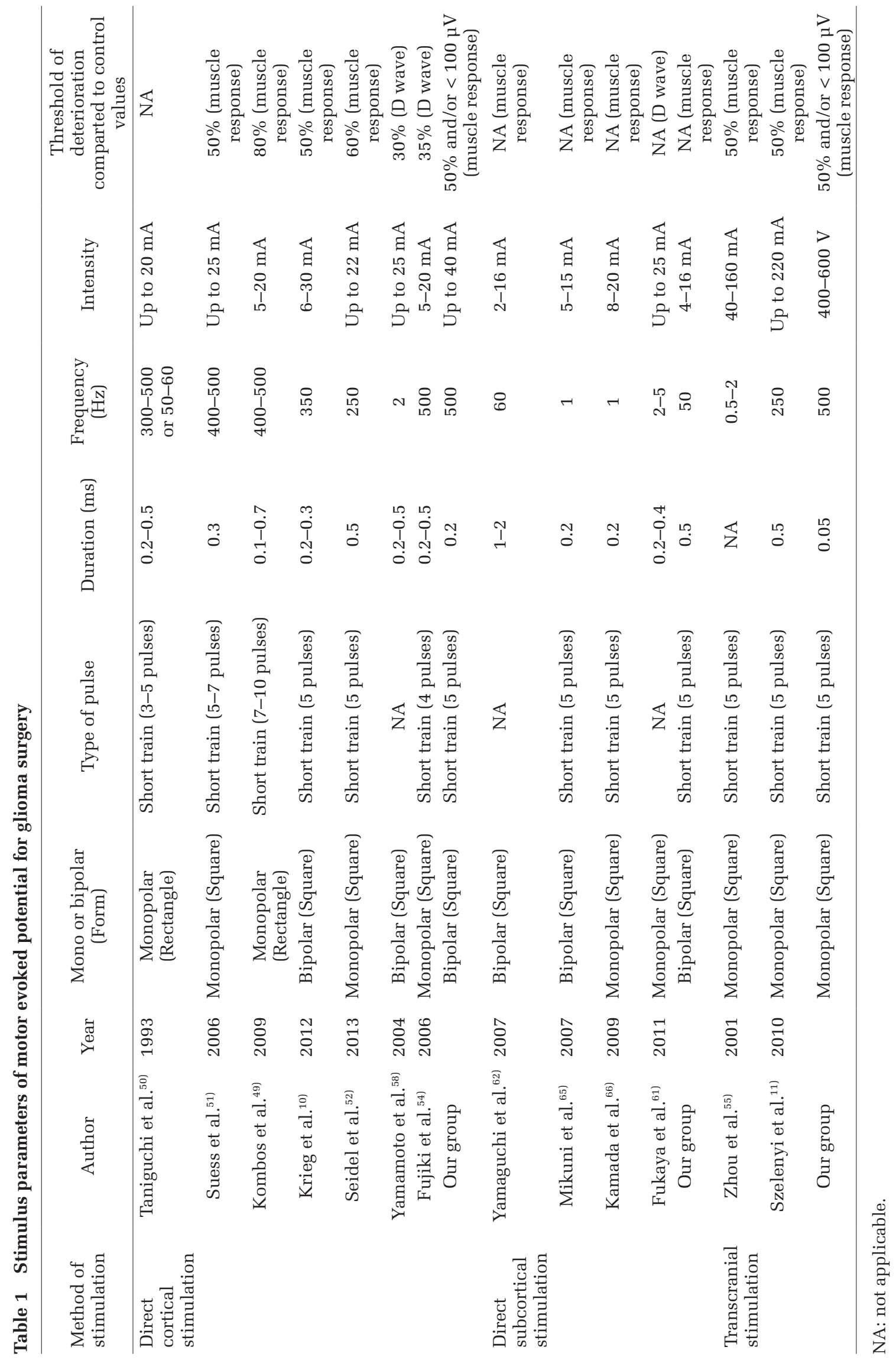

Neurol Med Chir (Tokyo) 55, January, 2015 
in amplitude of $50 \%{ }^{45,53-55)}$ We therefore adopted this criterion also. Furthermore, it should be kept in mind that the use of anesthesia significantly influences the reliability of CMAP monitoring, including inhalation anesthetics as well as residual muscle relaxants. Hence, anesthesia should be induced and maintained with continuous intravenous anesthetic administration (e.g., propofol).

DCS of the motor cortex can be used to record the MEP evoked from the epidural space in the spinal cord. ${ }^{56,57)}$ The corticospinal MEP response recorded using this procedure consists of a D-wave and a later I-wave. As described above, CMAP monitoring is significantly influenced by anesthesia, whereas the D-wave is resistant to the effects of anesthetics and muscle relaxants. Yamamoto et al. reported the availability of D-wave monitoring using bipolar stimulation with a multicontact plate electrode to preserve the motor function in 37 patients with glioma. ${ }^{58)}$ The authors identified that the critical point for eliciting a persistent motor disturbance was a $30 \%$ to $40 \%$ decrease in the amplitude of the $\mathrm{D}$-wave and decided to halt the tumor resection procedure before the decrease in amplitude reached $30 \%$; no permanent motor deficits were subsequently identified in their report.

In 1990, Berger et al. described a modification of the bipolar technique using a handheld probe already employed by Penfield. ${ }^{59)}$ This modification enables the application of intermittent direct electrical cortex stimulation, even during surgery under GA. Although this method does not permit the use of a qualitative analysis, this bipolar stimulation technique has since become the standard method for performing intraoperative cortical stimulation and mapping of motor areas under GA.

\section{Subcortical motor mapping with subcortical stimulation under GA}

Currently, subcortical mapping with a probe for bipolar and monopolar stimulation is used beyond cortical stimulation to identify the pyramidal tract. This technique has also been reported to be effective for glioma surgery. ${ }^{20,42,44,46)}$ Recently, Szelenyi et al. compared monopolar and bipolar stimulation aiming to identify the pyramidal tract. ${ }^{60)}$ The stimulation intensity (2-25 mA) for eliciting MEP was found to be significantly lower for monopolar multipulse stimulation. Therefore, the authors concluded that monopolar cathode stimulation is more effective for activating the subcortical region of the pyramidal tract than bipolar cathode stimulation. Fukaya et al. also reported that monopolar stimulation more clearly evokes subcortical D-waves from the spinal epidural space than bipolar stimulation. ${ }^{61)}$ In contrast,
Yamaguchi et al. developed a novel bipolar needle electrode that enables the identification of motor pathways following insertion of the device into the white matter and reported the efficacy of this instrument in preserving the motor function. ${ }^{62)}$

Subcortical mapping, in which the white matter is directly stimulated and the neural response is monitored, is the most reliable method for localizing functionally important white matter bundles, such as those in the pyramidal tract. However, subcortical mapping cannot be used to determine the distance and direction to the tract, although it does provide information as to whether the tract is near the stimulated position. With respect to visualizing the tract, the effectiveness of diffusion-weighted imaging (DWI) and diffusion-tensor imaging (DTI) techniques for fiber tracking has been investigated..$^{63,64)}$ Mikuni et al. directly compared the results of fiber tracking based on preoperative images and subcortical electrical stimulation during intraoperative neuronavigation in 40 patents with brain tumors located near the pyramidal tract. ${ }^{65)}$ Their results suggest that MEP are elicited from the subcortex when the distance between the stimulated subcortex and the estimated pyramidal tract on tractographyintegrated intraoperative neuronavigation is within $1 \mathrm{~cm}$. Kamada et al. also studied the association between the characteristics of the pyramidal tract on tractography and subcortical electrical stimulation and found that a minimum stimulus intensity of $20 \mathrm{~mA}, 15 \mathrm{~mA}, 10 \mathrm{~mA}$, and $5 \mathrm{~mA}$ was associated with stimulus points approximately $16 \mathrm{~mm}$, $13.2 \mathrm{~mm}, 9.6 \mathrm{~mm}$, and $4.8 \mathrm{~mm}$ from the pyramidal tract, respectively. ${ }^{66)}$ However, navigation with only preoperative images lacks precision, due to brain shift during tumor resection. In order to address this problem, we developed a neuronavigation system based on intraoperative DWI (iDWI), ${ }^{27,67)}$ while Prabhu et al. and Maesawa et al. examined the correlations between the results of subcortical stimulation and the course of the pyramidal tract on tractography using intraoperative DTI (iDTI) ${ }^{68,69)}$ We validated the bundle depicted on iDWI by considering the responses to subcortical stimulation (range: 4-16 $\mathrm{mA}$ ) and the distance between the site of stimulation and the depicted bundle (Fig. 3). Positive MEPs were detected in five of seven patients and the distance from the stimulation site to the depicted bundle was $0-4.7 \mathrm{~mm}$ (mean: \pm SD, $1.4 \pm 2.1 \mathrm{~mm}$ ). Negative (no) responses were obtained in all patients when the distance was greater than $5 \mathrm{~mm}$. These findings suggest that the white matter bundles depicted on iDWI may contain the pyramidal tract. Similarly, Prabhu et al. noted a trend toward worsening of neurological 


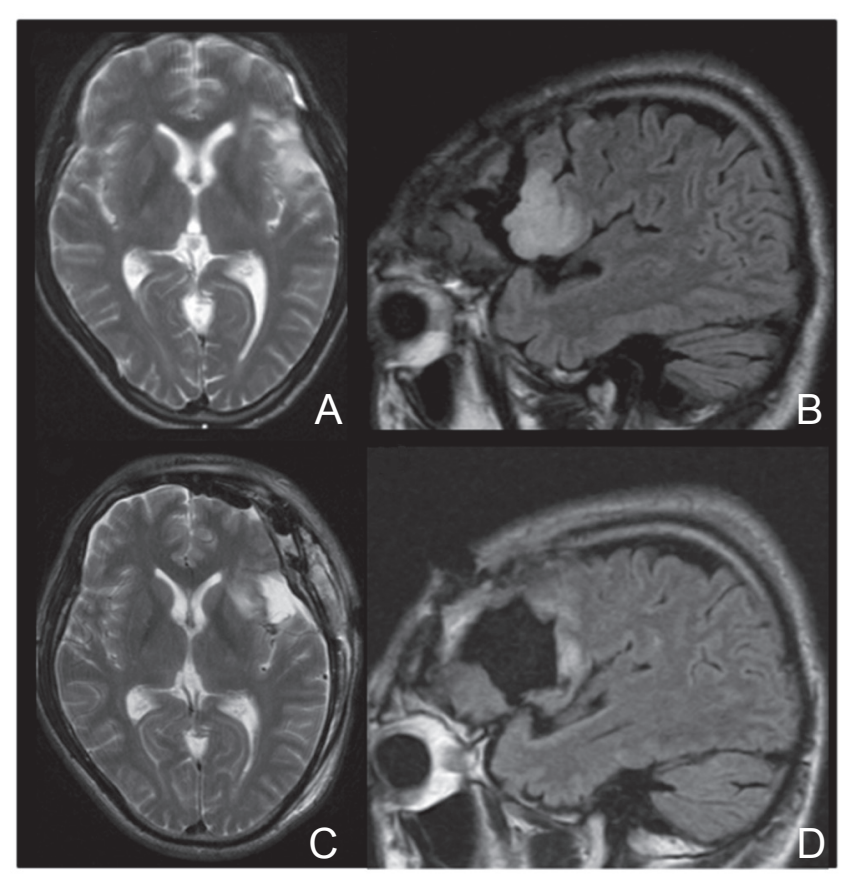

Fig. 3 A, B: Magnetic resonance (MR) images obtained before the second surgery demonstrating regrowth of the residual tumor just posterior to the prior resection cavity located in the frontal operculum. The tumor is hyperintense on axial $T_{2}$-weighted image $\left(T_{2} W I\right)$ (A) and sagittal fluid attenuated inversion recovery (FLAIR) image (B). C, D: MR images obtained after the second surgery showing that the tumor had been subtotally removed on $\mathrm{T}_{2}$ WI (C) and FLAIR (D).

deficits if the distance from the stimulus probe to the pyramidal tract is short $(<5 \mathrm{~mm})$, indicating the close proximity of the resection cavity to the pyramidal tract based on subcortical stimulation and iDTI tractography. ${ }^{69)}$ Meanwhile, Maesawa et al. demonstrated that the distance from intraoperative tractography of the pyramidal tract to the motor-evoked area exhibits a positive correlation with the intensity of stimulation. ${ }^{68)}$ However, the distance from preoperative tractography did not display a positive correlation with the stimulation intensity. These results indicate that intraoperative tractography and DWI show the location of the pyramidal tract more accurately than preoperative tractography. The combination of MEP monitoring and intraoperative tractography or DWI therefore enhances the quality of surgery for gliomas located in motor-eloquent areas.

\section{MEP monitoring with transcranial stimulation under GA}

The utility of MEP elicited by transcranial electric stimulation (TCS) during glioma surgery remains controversial. While, MEP monitoring by TCS has some advantages including that it can be compared with the contralateral MEP, it does not require the placement of a strip electrode which could injure the brain surface and/or cortical veins. Prior reports have studied MEP elicited by TCS using various stimulus parameters during brain tumor surgery (Table 1). Zhou and Kelly monitored the MEP elicited by TCS in 50 patients undergoing brain tumor resection. ${ }^{55)}$ The degree of postoperative worsening of the motor status was found to significantly correlate with the degree of reduction in the intraoperative MEP amplitude. The authors indicated that persistent intraoperative MEP reductions of more than $50 \%$ are associated with postoperative motor deficits. We also studied MEP using transcranial stimulation in 196 patients with brain tumors. In that study, monitoring of MEPs elicited by transcranial stimulation was successful in $98 \%$ of the patients. In 10 of 196 patients, we compared the findings of MEP elicited by TCS with DCS. In all patients, MEP alterations elicited by TCS were consistent with that obtained using DCS. These results support the efficacy of MEP monitoring using TCS during brain tumor surgery. In contrast, Li et al. reported that the MEP elicited by DCS, and not TCS, could be used to detect brain ischemia during brain tumor resection. ${ }^{70)}$ The authors thus insisted that MEP monitoring with DCS is superior to that of TCS in patients undergoing brain tumor resection. Recently, Szelenyi et al. demonstrated that MEP loss elicited by TCS bears a higher risk than MEP deterioration ( $>50 \%$ amplitude decrease and/or motor threshold increase) for postoperative motor deficits resulting from subcortical postoperative magnetic resonance changes in the pyramidal tract. ${ }^{11)}$ In contrast, the detection of MEP deterioration points to the presence of motor cortex lesions. Therefore, MEP deterioration should be considered as a warning sign during surgery performed close to the motor cortex. Although the availability of MEP monitoring with TCS remains controversial, we believe that the parallel use of TCS and DCS mutually improves the sensitivity of the intraoperative detection of motor impairment.

\section{Brain Functional Plasticity}

Low-grade gliomas are frequently located within or close to eloquent motor and language-related areas. Therefore, the EOR is limited by functional boundaries. Prior reports have suggested that slow-growing lesions, such as low-grade gliomas, induce functional reshaping due to plasticity. ${ }^{71-78)}$ These reports suggest that brain functional plasticity is induced by slow-growing lesions, as the 
slow time course of cerebral injury is a critical factor in neuroplasticity. For example, Robles et al. described two patients with low-grade gliomas located in the left dominant middle frontal gyrus who experienced language functional plasticity during the interval between two separate resection procedures. ${ }^{79)}$ The authors proposed that functional plasticity enables the surgeon to obtain an increased EOR during a second or even third surgery, while simultaneously facilitating preservation of the brain function in patients with low-grade gliomas located in eloquent areas. The following mechanisms of brain functional plasticity have been suggested by Robles et al.: (1) the slow infiltrative nature of low-grade glioma makes it possible to find functional areas within the tumor; (2) eloquent areas can be redistributed immediately around the tumor (perilesional plasticity); (3) other distant areas of the network within the same hemisphere can be activated; and (4) the contralateral homolog areas can also be recruited. ${ }^{79)}$ Recently, we reported a patient with oligoastrocytoma in the left inferior frontal gyrus in whom the functional plasticity of language was confirmed via intraoperative functional mapping and updated neuronavigation based on intraoperative MRI performed between two consecutive surgeries. ${ }^{80)}$ This case shows that language areas and related subcortical fibers cross the pre-central sulcus during tumor progression as a result of functional plasticity. Consequently, we were able to achieve subtotal removal of the recurrent tumor (Fig. 3). Most recently, Hayashi et al. described a functional shift in the motor area of a patient with an oligodendroglial tumor located in the right primary motor cortex. ${ }^{81)}$ The findings of intraoperative direct electrical stimulation under AC indicated that the motor region had shifted posteriorly and reorganized beyond the central sulcus. The results of these cases suggest that the second mechanism plays an important role in functional reorganization and contributes to maximal resection with functional preservation.

Several reports have suggested that brain function plasticity occurs preoperatively in patients with low-grade gliomas, based on the findings of functional magnetic resonance imaging (fMRI) and positron emission tomography (PET) ${ }^{82-88)}$ Prior fMRI and PET studies have demonstrated activation of motor and language areas within and around the tumor. ${ }^{89,90)}$ These reports indicate that the infiltrative characteristics of low-grade gliomas may make it possible for the neurologic function to persist within the tumor, while the slow rate of tumor invasion likely promotes functional plasticity.
Furthermore, activated areas have been recognized in distant areas within the same and contralateral hemisphere. ${ }^{90-92)}$ These phenomena suggest that slow-growing lesions affect functional networks and induce rewiring and the formation of new connections. These mechanisms may explain why most patients with low-grade gliomas have normal or only slightly impaired neurological outcomes.

Based on these findings, a multistage approach with intraoperative neurophysiological monitoring and AC for low-grade gliomas in eloquent areas is sustainable, while the combination of functional imaging and intraoperative electrical mapping can help to characterize brain functional plasticity. Additional studies with larger patient populations are needed to optimize this approach and validate treatment outcomes.

\section{Conclusion}

Intraoperative functional mapping and monitoring under AC have become increasingly widespread as a standard procedure for preserving the motor and language functions, maximizing the resection rate, and prolonging survival in glioma patients. On the other hand, less experienced facilities should seek to increase the learning curve for $\mathrm{AC}$ by visiting experienced facilities and referring to the appropriate guidelines. A facility authorization system and training courses have recently been developed by the Japan Neurosurgical Society and Japan Awake Surgery Society.

The new monitoring method using CCEP is feasible and useful for objectively evaluating the speech function, including direct assessments during AC. Intraoperative MEP monitoring during glioma resection under GA is also useful for maximizing the removal rate and minimizing neurological deficits by alarming that the touched area is close to eloquent motor areas and fibers.

Several papers have reported that brain function plasticity is induced during the interval between two surgeries for low-grade gliomas. This phenomenon indicates that functional plasticity may enable the surgeon to obtain an increased EOR during a second or even third surgery, while simultaneously facilitating preservation of the brain function in patients with low-grade gliomas located within eloquent areas.

\section{Acknowledgments}

This report was supported by Japan Science and Technology Agency, CREST. 


\section{Conflicts of Interest Disclosure}

No conflict of interest exists. All authors who are members of The Japan Neurosurgical Society (JNS) have registered online Self-reported COI Disclosure Statement Forms through the website for JNS members.

\section{References}

1) Stupp R, Hegi ME, Mason WP, van den Bent MJ, Taphoorn MJ, Janzer RC, Ludwin SK, Allgeier A, Fisher B, Belanger K, Hau P, Brandes AA, Gijtenbeek J, Marosi C, Vecht CJ, Mokhtari K, Wesseling P, Villa S, Eisenhauer E, Gorlia T, Weller M, Lacombe D, Cairncross JG, Mirimanoff RO; European Organisation for Research and Treatment of Cancer Brain Tumour and Radiation Oncology Groups; National Cancer Institute of Canada Clinical Trials Group: Effects of radiotherapy with concomitant and adjuvant temozolomide versus radiotherapy alone on survival in glioblastoma in a randomised phase III study: 5-year analysis of the EORTC-NCIC trial. Lancet Oncol 10: 459-466, 2009

2) Sabha N, Knobbe CB, Maganti M, Al Omar S, Bernstein M, Cairns R, Cako B, von Deimling A, Capper D, Mak TW, Kiehl TR, Carvalho P, Garrett E, Perry A, Zadeh G, Guha A, Croul S: Analysis of IDH mutation, 1p/19q deletion, and PTEN loss delineates prognosis in clinical low-grade diffuse gliomas. Neuro Oncol [Epub ahead of print], 2014

3) Soffietti R, Baumert BG, Bello L, von Deimling A, Duffau H, Frénay M, Grisold W, Grant R, Graus F, Hoang-Xuan K, Klein M, Melin B, Rees J, Siegal T, Smits A, Stupp R, Wick W; European Federation of Neurological Societies: Guidelines on management of low-grade gliomas: report of an EFNS-EANO Task Force. Eur J Neurol 17: 1124-1133, 2010

4) Wick W, Hartmann C, Engel C, Stoffels M, Felsberg J, Stockhammer F, Sabel MC, Koeppen S, Ketter R, Meyermann R, Rapp M, Meisner C, Kortmann RD, Pietsch T, Wiestler OD, Ernemann U, Bamberg M, Reifenberger G, von Deimling A, Weller M: NOA-04 randomized phase III trial of sequential radiochemotherapy of anaplastic glioma with procarbazine, lomustine, and vincristine or temozolomide. J Clin Oncol 27: 5874-5880, 2009

5) Lacroix M, Abi-Said D, Fourney DR, Gokaslan ZL, Shi W, DeMonte F, Lang FF, McCutcheon IE, Hassenbusch SJ, Holland E, Hess K, Michael C, Miller D, Sawaya R: A multivariate analysis of 416 patients with glioblastoma multiforme: prognosis, extent of resection, and survival. J Neurosurg 95: 190-198, 2001

6) Sanai N, Polley MY, McDermott MW, Parsa AT, Berger MS: An extent of resection threshold for newly diagnosed glioblastomas. J Neurosurg 115: 3-8, 2011

7) Smith JS, Chang EF, Lamborn KR, Chang SM, Prados MD, Cha S, Tihan T, Vandenberg S, McDermott MW, Berger MS: Role of extent of resection in the long- term outcome of low-grade hemispheric gliomas. $J$ Clin Oncol 26: 1338-1345, 2008

8) Nitta M, Muragaki Y, Maruyama T, Iseki H, Ikuta S, Konishi Y, Saito T, Tamura M, Chernov M, Watanabe A, Okamoto S, Maebayashi K, Mitsuhashi N, Okada Y: Updated therapeutic strategy for adult low-grade glioma stratified by resection and tumor subtype. Neurol Med Chir (Tokyo) 53: 447-454, 2013

9) Deletis V, Camargo AB: Transcranial electrical motor evoked potential monitoring for brain tumor resection. Neurosurgery 49: 1488-1489, 2001

10) Krieg SM, Shiban E, Droese D, Gempt J, Buchmann N, Pape H, Ryang YM, Meyer B, Ringel F: Predictive value and safety of intraoperative neurophysiological monitoring with motor evoked potentials in glioma surgery. Neurosurgery 70: 1060-1070; discussion 1070-1071, 2012

11) Szelényi A, Hattingen E, Weidauer S, Seifert V, Ziemann U: Intraoperative motor evoked potential alteration in intracranial tumor surgery and its relation to signal alteration in postoperative magnetic resonance imaging. Neurosurgery 67: 302-313, 2010

12) Grant GA, Farrell D, Silbergeld DL: Continuous somatosensory evoked potential monitoring during brain tumor resection. Report of four cases and review of the literature. $J$ Neurosurg 97: 709-713, 2002

13) Cedzich C, Taniguchi M, Schäfer S, Schramm J: Somatosensory evoked potential phase reversal and direct motor cortex stimulation during surgery in and around the central region. Neurosurgery 38: 962-970, 1996

14) Chacko AG, Thomas SG, Babu KS, Daniel RT, Chacko G, Prabhu K, Cherian V, Korula G: Awake craniotomy and electrophysiological mapping for eloquent area tumours. Clin Neurol Neurosurg 115: 329-334, 2013

15) Lüders H, Awad I, Burgess R, Wyllie E, Van Ness P: Subdural electrodes in the presurgical evaluation for surgery of epilepsy. Epilepsy Res Suppl 5: 147-156, 1992

16) De Witt Hamer PC, Robles SG, Zwinderman AH, Duffau H, Berger MS: Impact of intraoperative stimulation brain mapping on glioma surgery outcome: a meta-analysis. J Clin Oncol 30: 25592565, 2012

17) Brown T, Shah AH, Bregy A, Shah NH, Thambuswamy M, Barbarite E, Fuhrman T, Komotar RJ: Awake craniotomy for brain tumor resection: the rule rather than the exception? J Neurosurg Anesthesiol 25: 240-247, 2013

18) Kayama T; Guidelines committee of the Japan awake surgery conference: The guidelines for awake craniotomy guidelines committee of the Japan awake surgery conference. Neurol Med Chir (Tokyo) 52: 119-141, 2012

19) Kinno R, Muragaki $Y$, Hori T, Maruyama T, Kawamura M, Sakai KL: Agrammatic comprehension caused by a glioma in the left frontal cortex. Brain Lang 110: 71-80, 2009 
20) Duffau H: Contribution of cortical and subcortical electrostimulation in brain glioma surgery: methodological and functional considerations. Neurophysiol Clin 37: 373-382, 2007

21) Kim SS, McCutcheon IE, Suki D, Weinberg JS, Sawaya R, Lang FF, Ferson D, Heimberger AB, DeMonte F, Prabhu SS: Awake craniotomy for brain tumors near eloquent cortex: correlation of intraoperative cortical mapping with neurological outcomes in 309 consecutive patients. Neurosurgery 64: 836-845; discussion 345-846, 2009

22) Mikuni N, Miyamoto S: Surgical treatment for glioma: extent of resection applying functional neurosurgery. Neurol Med Chir (Tokyo) 50: 720-726, 2010

23) Sanai N, Mirzadeh Z, Berger MS: Functional outcome after language mapping for glioma resection. $N$ Engl J Med 358: 18-27, 2008

24) Yoshimitsu K, Maruyama T, Muragaki Y, Suzuki T, Saito T, Nitta M, Tanaka M, Chernov M, Tamura M, Ikuta S, Okamoto J, Okada Y, Iseki H: Wireless modification of the intraoperative examination monitor for awake surgery. Neurol Med Chir (Tokyo) 51: 472-476, 2011

25) Yoshimitsu K, Suzuki T, Muragaki Y, Chernov M, Iseki $\mathrm{H}$ : Development of modified intraoperative examination monitor for awake surgery (IEMAS) system for awake craniotomy during brain tumor resection. Conf Proc IEEE Eng Med Biol Soc 2010: 6050-6053, 2010

26) Muragaki $Y$, Iseki $H$, Maruyama $T$, Tanaka $M$, Shinohara C, Suzuki T, Yoshimitsu K, Ikuta S, Hayashi M, Chernov M, Hori T, Okada Y, Takakura $\mathrm{K}$ : Information-guided surgical management of gliomas using low-field-strength intraoperative MRI. Acta Neurochir Suppl 109: 67-72, 2011

27) Ozawa N, Muragaki Y, Nakamura R, Iseki H: Identification of the pyramidal tract by neuronavigation based on intraoperative diffusion-weighted imaging combined with subcortical stimulation. Stereotact Funct Neurosurg 87: 18-24, 2009

28) Trinh VT, Fahim DK, Shah K, Tummala S, McCutcheon IE, Sawaya R, Suki D, Prabhu SS: Subcortical injury is an independent predictor of worsening neurological deficits following awake craniotomy procedures. Neurosurgery 72: 160-169, 2013

29) Szelenyi A, Bello L, Duffau H, Fava E, Feigl GC, Galanda M, Neuloh G, Signorelli F, Sala F, Workgroup for Intraoperative Management in Low-Grade Glioma Surgery within the European Low-Grade Glioma N: Intraoperative electrical stimulation in awake craniotomy: methodological aspects of current practice. Neurosurg Focus 28: E7, 2010

30) Muragaki Y, Iseki H, Takakura K, Maruyama T, Hori $\mathrm{T}$ : [Awake craniotomy and functional mapping for surgery of brain tumors]. Nihon Rinsho 63(Suppl 9): 330-340, 2005 (Japanese)

31) Suzuki K, Mikami T, Sugino T, Wanibuchi M, Miyamoto S, Hashimoto N, Mikuni N: Discrepancy between voluntary movement and motor-evoked potentials in evaluation of motor function during clipping of anterior circulation aneurysms. World Neurosurg [Epub ahead of print], 2013

32) Conner CR, Ellmore TM, DiSano MA, Pieters TA, Potter AW, Tandon N: Anatomic and electrophysiologic connectivity of the language system: a combined DTI-CCEP study. Comput Biol Med 41: 1100-1109, 2011

33) Enatsu R, Kubota Y, Kakisaka Y, Bulacio J, Piao Z, O'Connor T, Horning K, Mosher J, Burgess RC, Bingaman W, Nair DR: Reorganization of posterior language area in temporal lobe epilepsy: a corticocortical evoked potential study. Epilepsy Res 103: 73-82, 2013

34) Enatsu R, Matsumoto R, Piao Z, O'Connor T, Horning K, Burgess RC, Bulacio J, Bingaman W, Nair DR: Cortical negative motor network in comparison with sensorimotor network: a cortico-cortical evoked potential study. Cortex 49: 2080-2096, 2013

35) Kubota Y, Enatsu R, Gonzalez-Martinez J, Bulacio J, Mosher J, Burgess RC, Nair DR: In vivo human hippocampal cingulate connectivity: a corticocortical evoked potentials (CCEPs) study. Clin Neurophysiol 124: 1547-1556, 2013

36) Matsumoto R, Nair DR, LaPresto E, Bingaman W, Shibasaki H, Lüders HO: Functional connectivity in human cortical motor system: a cortico-cortical evoked potential study. Brain 130: 181-197, 2007

37) Matsumoto R, Nair DR, LaPresto E, Najm I, Bingaman W, Shibasaki H, Lüders HO: Functional connectivity in the human language system: a cortico-cortical evoked potential study. Brain 127: 2316-2330, 2004

38) Saito T, Tamura M, Muragaki Y, Maruyama T, Kubota Y, Fukuchi S, Nitta M, Chernov M, Okamoto S, Sugiyama K, Kurisu K, Sakai KL, Okada Y, Iseki $\mathrm{H}$ : Intraoperative cortico-cortical evoked potentials for the evaluation of language function during brain tumor resection: initial experience with 13 cases. J Neurosurg 121: 827-838, 2014

39) Yamao $Y$, Matsumoto R, Kunieda T, Arakawa Y, Kobayashi K, Usami K, Shibata S, Kikuchi T, Sawamoto N, Mikuni N, Ikeda A, Fukuyama H, Miyamoto S: Intraoperative dorsal language network mapping by using single-pulse electrical stimulation. Hum Brain Mapp 35: 4345-6143, 2014

40) Gil Robles S, Gatignol P, Capelle L, Mitchell MC, Duffau $\mathrm{H}$ : The role of dominant striatum in language: a study using intraoperative electrical stimulations. J Neurol Neurosurg Psychiatr 76: 940-946, 2005

41) Ebeling U, Schmid UD, Ying H, Reulen HJ: Safe surgery of lesions near the motor cortex using intra-operative mapping techniques: a report on 50 patients. Acta Neurochir (Wien) 119: 23-28, 1992

42) Neuloh G, Pechstein U, Schramm J: Motor tract monitoring during insular glioma surgery. J Neurosurg 106: 582-592, 2007

43) Yingling CD, Ojemann S, Dodson B, Harrington MJ, Berger MS: Identification of motor pathways during tumor surgery facilitated by multichannel electromyographic recording. J Neurosurg 91: 922-927, 1999 
44) Neuloh G, Pechstein U, Cedzich C, Schramm J: Motor evoked potential monitoring with supratentorial surgery. Neurosurgery 61: 337-346; discussion 346-348, 2007

45) Sala F, Lanteri P: Brain surgery in motor areas: the invaluable assistance of intraoperative neurophysiological monitoring. J Neurosurg Sci 47: 79-88, 2003

46) Berger MS, Hadjipanayis CG: Surgery of intrinsic cerebral tumors. Neurosurgery 61: 279-304; discussion 304-305, 2007

47) Kombos T, Süss O, Vajkoczy P: Subcortical mapping and monitoring during insular tumor surgery. Neurosurg Focus 27: E5, 2009

48) Gempt J, Krieg SM, Hüttinger S, Buchmann N, Ryang YM, Shiban E, Meyer B, Zimmer C, Förschler A, Ringel F: Postoperative ischemic changes after glioma resection identified by diffusion-weighted magnetic resonance imaging and their association with intraoperative motor evoked potentials. $J$ Neurosurg 119: 829-836, 2013

49) Kombos T, Picht T, Derdilopoulos A, Suess O: Impact of intraoperative neurophysiological monitoring on surgery of high-grade gliomas. J Clin Neurophysiol 26: 422-425, 2009

50) Taniguchi M, Cedzich C, Schramm J: Modification of cortical stimulation for motor evoked potentials under general anesthesia: technical description. Neurosurgery 32: 219-226, 1993

51) Suess O, Suess S, Brock M, Kombos T: Intraoperative electrocortical stimulation of Brodman area 4: a 10-year analysis of 255 cases. Head Face Med 2: 20,2006

52) Seidel K, Beck J, Stieglitz L, Schucht P, Raabe A: The warning-sign hierarchy between quantitative subcortical motor mapping and continuous motor evoked potential monitoring during resection of supratentorial brain tumors. J Neurosurg 118: 287-296, 2013

53) Neuloh G, Pechstein U, Cedzich C, Schramm J: Motor evoked potential monitoring with supratentorial surgery. Neurosurgery 54: 1061-1070; discussion 1070-1072, 2004

54) Fujiki M, Furukawa Y, Kamida T, Anan M, Inoue R, Abe T, Kobayashi H: Intraoperative corticomuscular motor evoked potentials for evaluation of motor function: a comparison with corticospinal D and I waves. J Neurosurg 104: 85-92, 2006

55) Zhou HH, Kelly PJ: Transcranial electrical motor evoked potential monitoring for brain tumor resection. Neurosurgery 48: 1075-1080; discussion 1080-1081, 2001

56) Katayama Y, Tsubokawa T, Maejima S, Hirayama T, Yamamoto T: Corticospinal direct response in humans: identification of the motor cortex during intracranial surgery under general anaesthesia. $J$ Neurol Neurosurg Psychiatr 51: 50-59, 1988

57) Yamamoto T, Xing JA, Katayama Y, Tsubokawa T, Hirayama T, Maejima S: Spinal cord responses to feline transcranial brain stimulation: evidence for involvement of cerebellar pathways. J Neurotrauma 7: 247-256, 1990

58) Yamamoto T, Katayama Y, Nagaoka T, Kobayashi K, Fukaya C: Intraoperative monitoring of the corticospinal motor evoked potential (D-wave): clinical index for postoperative motor function and functional recovery. Neurol Med Chir (Tokyo) 44: 170-180; discussion 181-182, 2004

59) Berger MS, Ojemann GA, Lettich E: Neurophysiological monitoring during astrocytoma surgery. Neurosurg Clin N Am 1: 65-80, 1990

60) Szelényi A, Senft C, Jardan M, Forster MT, Franz $\mathrm{K}$, Seifert V, Vatter H: Intra-operative subcortical electrical stimulation: a comparison of two methods. Clin Neurophysiol 122: 1470-1475, 2011

61) Fukaya C, Sumi K, Otaka T, Shijo K, Nagaoaka T, Kobayashi K, Oshima H, Watanabe T, Yamamoto T, Katayama Y: Corticospinal descending direct wave elicited by subcortical stimulation. J Clin Neurophysiol 28: 297-301, 2011

62) Yamaguchi F, Takahashi H, Teramoto A: Intraoperative detection of motor pathways using a simple electrode provides safe brain tumor surgery. J Clin Neurosci 14: 1106-1110, 2007

63) Basser PJ, Pajevic S, Pierpaoli C, Duda J, Aldroubi A: In vivo fiber tractography using DT-MRI data. Magn Reson Med 44: 625-632, 2000

64) Krings T, Reinges MH, Thiex R, Gilsbach JM, Thron A: Functional and diffusion-weighted magnetic resonance images of space-occupying lesions affecting the motor system: imaging the motor cortex and pyramidal tracts. J Neurosurg 95: 816-824, 2001

65) Mikuni N, Okada T, Enatsu R, Miki Y, Hanakawa T, Urayama S, Kikuta K, Takahashi JA, Nozaki K, Fukuyama H, Hashimoto N: Clinical impact of integrated functional neuronavigation and subcortical electrical stimulation to preserve motor function during resection of brain tumors. J Neurosurg 106: 593-598, 2007

66) Kamada K, Todo T, Ota T, Ino K, Masutani Y, Aoki S, Takeuchi F, Kawai K, Saito N: The motor-evoked potential threshold evaluated by tractography and electrical stimulation. J Neurosurg 111: 785-795, 2009

67) Ozawa N, Muragaki Y, Nakamura R, Hori T, Iseki $\mathrm{H}$ : Shift of the pyramidal tract during resection of the intraaxial brain tumors estimated by intraoperative diffusion-weighted imaging. Neurol Med Chir (Tokyo) 49: 51-56, 2009

68) Maesawa S, Fujii M, Nakahara N, Watanabe T, Wakabayashi T, Yoshida J: Intraoperative tractography and motor evoked potential (MEP) monitoring in surgery for gliomas around the corticospinal tract. World Neurosurg 74: 153-161, 2010

69) Prabhu SS, Gasco J, Tummala S, Weinberg JS, Rao G: Intraoperative magnetic resonance imagingguided tractography with integrated monopolar subcortical functional mapping for resection of brain tumors. Clinical article. J Neurosurg 114: 719-726, 2011 
70) Li F, Deshaies EM, Allott G, Canute G, Gorji R: Direct cortical stimulation but not transcranial electrical stimulation motor evoked potentials detect brain ischemia during brain tumor resection. Am J Electroneurodiagnostic Technol 51: 191-197, 2011

71) Benzagmout M, Gatignol P, Duffau H: Resection of World Health Organization Grade II gliomas involving Broca's area: methodological and functional considerations. Neurosurgery 61: 741-752; discussion 752-753, 2007

72) Desmurget M, Bonnetblanc F, Duffau H: Contrasting acute and slow-growing lesions: a new door to brain plasticity. Brain 130: 898-914, 2007

73) Duffau H, Lopes M, Arthuis F, Bitar A, Sichez JP, Van Effenterre R, Capelle L: Contribution of intraoperative electrical stimulations in surgery of low grade gliomas: a comparative study between two series without (1985-96) and with (1996-2003) functional mapping in the same institution. J Neurol Neurosurg Psychiatr 76: 845-851, 2005

74) Duffau H: New concepts in surgery of WHO grade II gliomas: functional brain mapping, connectionism and plasticity-a review. J Neurooncol 79: 77-115, 2006

75) Duffau H: Introduction. Surgery of gliomas in eloquent areas: from brain hodotopy and plasticity to functional neurooncology. Neurosurg Focus 28: Intro, 2010

76) Duffau H: The challenge to remove diffuse lowgrade gliomas while preserving brain functions. Acta Neurochir (Wien) 154: 569-574, 2012

77) Duffau H, Denvil D, Capelle L: Long term reshaping of language, sensory, and motor maps after glioma resection: a new parameter to integrate in the surgical strategy. J Neurol Neurosurg Psychiatr 72: 511-516, 2002

78) Martino J, Taillandier L, Moritz-Gasser S, Gatignol P, Duffau H: Re-operation is a safe and effective therapeutic strategy in recurrent WHO grade II gliomas within eloquent areas. Acta Neurochir (Wien) 151: 427-436; discussion 436, 2009

79) Robles SG, Gatignol P, Lehéricy S, Duffau H: Longterm brain plasticity allowing a multistage surgical approach to World Health Organization Grade II gliomas in eloquent areas. J Neurosurg 109: 615-624, 2008

80) Saito T, Muragaki Y, Miura I, Tamura M, Maruyama T, Nitta M, Kurisu K, Iseki H, Okada Y: Functional plasticity of language confirmed with intraoperative electrical stimulations and updated neuronavigation: case report of low-grade glioma of the left inferior frontal gyrus. Neurol Med Chir (Tokyo) 54: 587-592, 2014

81) Hayashi Y, Nakada M, Kinoshita M, Hamada JI: Functional reorganization in the patient with progressing glioma of pure primary motor cortex: a case report with special reference to the topographic central sulcus defined by somatosensoryevoked potential. World Neurosurg [Epub ahead of print], 2013
82) Hayashi Y, Nakada M, Kinoshita M, Hamada J: Surgical strategies for nonenhancing slow-growing gliomas with special reference to functional reorganization: review with own experience. Neurol Med Chir (Tokyo) 53: 438-446, 2013

83) Ius T, Angelini E, Thiebaut de Schotten M, Mandonnet E, Duffau H: Evidence for potentials and limitations of brain plasticity using an atlas of functional resectability of WHO grade II gliomas: towards a "minimal common brain”. Neuroimage 56: 992-1000, 2011

84) Rosenberg K, Liebling R, Avidan G, Perry D, SimanTov T, Andelman F, Ram Z, Fried I, Hendler T: Language related reorganization in adult brain with slow growing glioma: fMRI prospective case-study. Neurocase 14: 465-473, 2008

85) Saur D, Lange R, Baumgaertner A, Schraknepper V, Willmes K, Rijntjes M, Weiller C: Dynamics of language reorganization after stroke. Brain 129: 1371-1384, 2006

86) Staudt M: Reorganization after pre- and perinatal brain lesions. J Anat 217: 469-474, 2010

87) Thiel A, Habedank B, Herholz K, Kessler J, Winhuisen L, Haupt WF, Heiss WD: From the left to the right: How the brain compensates progressive loss of language function. Brain Lang 98: 57-65, 2006

88) Thiel A, Herholz K, Koyuncu A, Ghaemi M, Kracht LW, Habedank B, Heiss WD: Plasticity of language networks in patients with brain tumors: a positron emission tomography activation study. Ann Neurol 50: 620-629, 2001

89) Krainik A, Lehéricy S, Duffau H, Capelle L, Chainay H, Cornu P, Cohen L, Boch AL, Mangin JF, Le Bihan D, Marsault C: Postoperative speech disorder after medial frontal surgery: role of the supplementary motor area. Neurology 60: 587-594, 2003

90) Meyer PT, Sturz L, Sabri O, Schreckenberger M, Spetzger U, Setani KS, Kaiser HJ, Buell U: Preoperative motor system brain mapping using positron emission tomography and statistical parametric mapping: hints on cortical reorganisation. J Neurol Neurosurg Psychiatr 74: 471-478, 2003

91) Holodny AI, Schulder M, Ybasco A, Liu WC: Translocation of Broca's area to the contralateral hemisphere as the result of the growth of a left inferior frontal glioma. J Comput Assist Tomogr 26: 941-943, 2002

92) Krainik A, Duffau H, Capelle L, Cornu P, Boch AL, Mangin JF, Le Bihan D, Marsault C, Chiras J, Lehéricy S: Role of the healthy hemisphere in recovery after resection of the supplementary motor area. Neurology 62: 1323-1332, 2004

Address reprint requests to: Yoshihiro Muragaki, MD, $\mathrm{PhD}$, Faculty of Advanced Techno-Surgery, Institute of Advanced Biomedical Engineering and Science, Tokyo Women's Medical University, 8-1 Kawada-cho, Shinjuku-ku, Tokyo 162-8666, Japan. e-mail: ymuragaki@twmu.ac.jp 